\title{
Directional Coupler Biosensor with Molecularly Imprinted Polymer
}

\author{
Ken Uchiyamada, ${ }^{1}$ Kyohei Okubo, ${ }^{2}$ Kiyoshi Asakawa, ${ }^{1}$ Yuri Kamon, ${ }^{3}$ \\ Yukiya Kitayama, ${ }^{3}$ Toshifumi Takeuchi, ${ }^{3}$ and Hiroaki Suzuki ${ }^{*}$ \\ ${ }^{1}$ Graduate School of Pure and Applied Sciences, University of Tsukuba, \\ 1-1-1 Tennodai, Tsukuba, Ibaraki 305-8573, Japan \\ ${ }^{2}$ School of Engineering, The University of Tokyo 2-11-16 Yayoi, Bunkyo, Tokyo 113-8656, Japan. \\ ${ }^{3}$ Graduate School of Engineering, Kobe University, 1-1 Rokkodai-cho, Nada-ku, Kobe 657-8501, Japan
}

(Received November 24, 2017; accepted January 4, 2018)

Keywords: directional coupler, silicon nitride waveguide, molecularly imprinted polymer (MIP), human serum albumin (HSA)

A microfabricated directional coupler (DC) was used for the label-free, real-time detection of proteins. As an alternative to biomolecules such as antibodies, molecularly imprinted polymer (MIP) was used. With human serum albumin (HSA) as a model analyte, distinct responses were observed. On the other hand, the sensor did not respond to bovine serum albumin (BSA). Also, with a nonimprinted polymer (NIP), no responses were observed for HSA. The MIP could be regenerated, and responses of the same sensor after three cycles of measurement and regeneration gave similar values.

\section{Introduction}

Various biosensors have been developed on the basis of electrochemical and optical detection principles and with a variety of materials for sensing. ${ }^{(1-4)}$ Among them, photonic biosensors have attracted attention because of their high sensitivity, nondestructiveness, and real-time sensing. ${ }^{(5,6)}$ In particular, interferometric sensors based on Mach-Zehnder ${ }^{(7)}$ and Young ${ }^{(8)}$ interferometers feature the possibility of miniaturization and integration with other components such as microfluidic systems. ${ }^{(9,10)}$

In affinity biosensing, the most critical part is the interaction between target molecules and ligands. However, one problem with biomacromolecules such as antibodies is the irreversibility of reaction, loss of activity, time-consuming production and resulting high cost, and difficulty of quality control. In particular, the irreversible binding of molecules often forces the device to be disposable. To overcome these problems, alternative artificial materials have been developed. Among them, molecularly imprinted polymer (MIP) has been attracting attention. Molecular imprinting is a technique of making molecular templates by copolymerization of target molecules (template), functional monomers that bind to the target molecules covalently or noncovalently, and cross-linkers. After removing the template molecules, imprinted cavities ${ }^{*}$ Corresponding author: e-mail: hsuzuki@ims.tsukuba.ac.jp http://dx.doi.org/10.18494/SAM.2018.1815 
are formed in the polymer matrix (Fig. 1). The cavities that are complementary to the template molecules in shape and size recognize the target molecules and bind them. ${ }^{(11-13)}$

Previously, we reported a biosensor based on a directional coupler (DC). The device could detect a very slight change in refractive index, $n$, and achieved a detection limit of $10 \mathrm{nM}$ for DNA and $170 \mathrm{nM}$ for streptavidin. ${ }^{(14,15)}$ On the basis of the previous success, we used the DC sensor coupled with MIP and attempted the detection of human serum albumin (HSA), which is one of the most common proteins in the human body ( $70 \%$ of the protein in human serum) and is used as a marker protein for liver disease, thyroid disease, and cancer, for example. ${ }^{(16)}$ Compared with natural antibodies, an advantage of MIP is regeneration after the binding of proteins and repeated use. ${ }^{(17)}$ We also demonstrate that this was actually the case with our DC sensor.

\section{Experimental Procedure}

\subsection{Reagents and materials}

Silicon wafers ( 2 in., $280 \mu \mathrm{m}$ thick) with a silicon nitride ( $\mathrm{SiN}$ ) layer (300 $\mathrm{nm}$ thick, $n=$ 2.00 at $1.31 \mu \mathrm{m}$ wavelength) and an underlying $\mathrm{SiO}_{2}$ layer $(2 \mu \mathrm{m}$ thick, $n=1.44$ at $1.31 \mu \mathrm{m}$ wavelength) were obtained from the Research Institute for Nano-device and Biosystems at Hiroshima University. The $\mathrm{SiO}_{2}$ layers were thermally grown at $1000{ }^{\circ} \mathrm{C}$ and the $\mathrm{SiN}$ layer was deposited by low-pressure chemical vapor deposition (LPCVD) in a vertical thermal reactor with a gaseous mixture of $\mathrm{SiH}_{2} \mathrm{Cl}_{2}$ and $\mathrm{NH}_{3}$ (pressure: 0.16 Torr) at $750{ }^{\circ} \mathrm{C}$. The LPCVD SiN film features low stress and low hydrogen content. ${ }^{(18)}$

Other materials used for the fabrication and characterization of the device were obtained from the following commercial sources: a thick-film photoresist SU-8 25 from MicroChem (Newton, MA, USA); prepolymer solution of poly(dimethylsiloxane) (PDMS) (KE-1300T) from Shin-Etsu Chemical (Tokyo, Japan); glucose, human serum albumin (HSA), bovine serum albumin (BSA) and sodium dodecyl sulfate (SDS) from Wako Pure Chemical Industries (Osaka, Japan); 2-methacryloyloxyethyl phosphprylcholine (MPC), $N, N^{\prime}$-methylenebisacrylamide (MBAA), and bipyridine (Bpy) from Tokyo Chemical Industry (Tokyo, Japan); 1-ethyl-3-

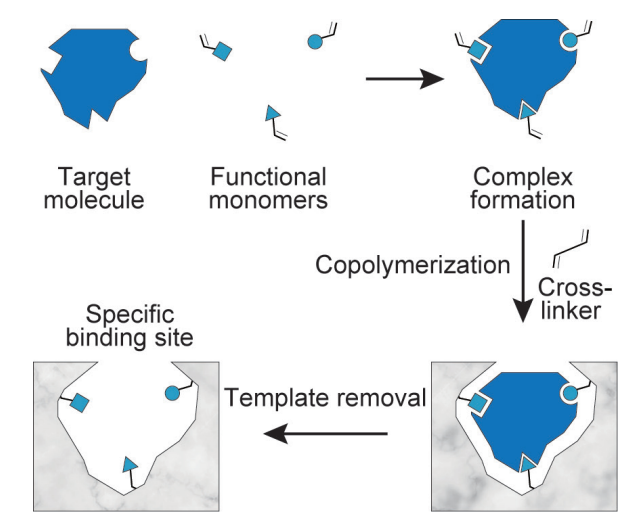

Fig. 1. (Color online) Scheme of molecular imprinting. 
(3-dimethylaminopropyl) carbodiimide hydrochloride (EDS $\cdot \mathrm{HCl})$ from Watanabe Chemical Industries (Hiroshima, Japan); $N$-hydroxysuccinimide (NHS) from Sigma-Aldrich Japan (Tokyo, Japan); positive electron beam (EB) resist gL-2000 from Gluon Lab (Tokyo, Japan). Other chemicals were of analytical reagent grade and were used as received. All sample and buffer solutions were prepared with deionized (DI) water obtained from a water purification system (Direct-Q UV 3, Merck, Darmstadt, Germany).

\subsection{Fabrication of DC sensor}

Figure 2(a) shows the structure and construction of the DC sensor. The structure was formed with a silicon substrate with $2-\mu \mathrm{m}$-thick silicon oxide as a cladding layer and a $300-\mathrm{nm}$ thick silicon nitride layer to form waveguides. DC sensors with 400-nm-wide and 300-nmhigh waveguides were formed on a chip of $18 \times 10 \mathrm{~mm}^{2}$. To form the sensing region of the DC sensor, two single-mode SiN parallel waveguides were separated with a 150-nm-wide gap. The angle between the connection of the sensing region and both input and output waveguides was $5^{\circ}$. The length of the sensing region was $406 \mu \mathrm{m}$. To block unnecessary background signals from the unetched parts of the SiN layer that functions as a slab waveguide, 1- $\mu$ m-wide slits were formed near the input and output waveguide regions [Fig. 2 (b)]. A PDMS substrate with a flow channel was placed on the DC sensor.

To fabricate the device, a 40-nm-thick aluminum layer was formed on the SiN film by sputtering (CSF-4EP-LL, Shibaura Mechatronics). The positive EB resist (gL-2000, $400 \mathrm{~nm}$

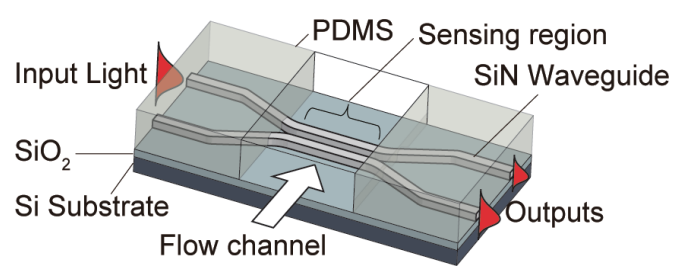

(a)

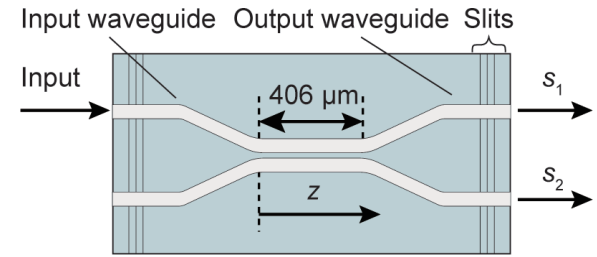

(b)

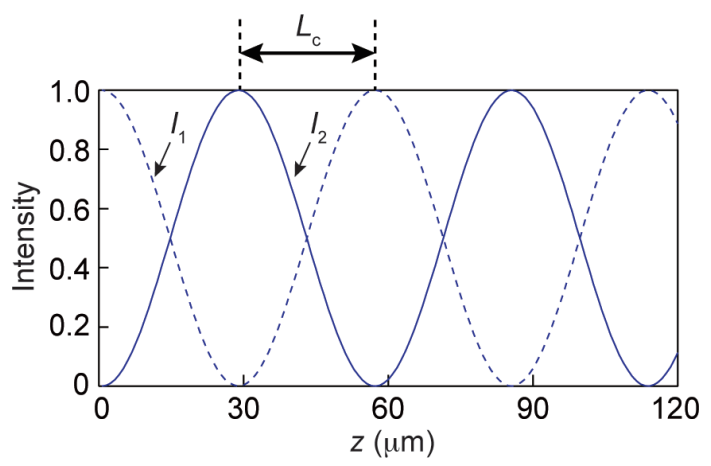

(c)

Fig. 2. (Color online) (a) Structure of the DC sensor with a microfluidic channel used for the transducer. (b) Top view of the SiN DC sensor. (c) Optical power distribution along the direction of light propagation $z$. The diagram shows the complete coupling length $L_{c}$, which is defined as the distance at which the optical power transfers completely from one waveguide to the other. 
thick) was then spin-coated, followed by prebaking for 5 min at $180{ }^{\circ} \mathrm{C}$. The EB resist was exposed to an electron beam (125 kV) using an EB lithography system (ELS-F125N, Elionix) with a dose of $300 \mu \mathrm{C} / \mathrm{cm}^{2}$. The EB resist was developed in xylene and postbaked for $3 \mathrm{~min}$ at $140{ }^{\circ} \mathrm{C}$. The aluminum layer was etched by reactive ion etching (RIE) (RIE-101iPH, Samco) in $\mathrm{BCl}_{3}$ plasma for $3 \mathrm{~min}$ at a pressure of $5 \mathrm{~Pa}$ and $\mathrm{RF}$ power of $200 \mathrm{~W}$. The SiN layer was etched by RIE (RIE-10N, Samco) in a $\mathrm{CHF}_{3}$ plasma for $16.5 \mathrm{~min}$ at a pressure of $0.5 \mathrm{~Pa}$ and a $\mathrm{RF}$ power of $50 \mathrm{~W}$. The resist was removed using an oxygen plasma cleaner (PR500, Yamato Scientific) for $10 \mathrm{~min}$ at $200 \mathrm{~Pa}$ and $300 \mathrm{~W}$. The aluminum was removed in a $3 \mathrm{M} \mathrm{HCl}$ solution. For measurements, the chips were cleaved to expose the output and input facets at the edges of the waveguides.

\subsection{Formation of the MIP}

The SiN surface of the sensing region was modified with a 10-nm-thick layer of MIP, nonimprinted polymer (NIP), or MPC following the protocol of Inoue et al. ${ }^{(19)}$ The polymer layers were formed by atom transfer radical polymerization using a surface-initiated activator generated by electron transfer (SI-AGET ATRP). ${ }^{(20)}$ MIP was prepared with pyrrolidyl acrylate (PyA) as the functional monomer, MPC as the comonomer for protection against nonspecific binding of other proteins, and a hydrophilic cross-linker, MBAA. MPC reduces nonspecific binding of proteins. ${ }^{(21)}$ PyA binds to HSA by electrostatic interaction between the plus charges of the pyrrolidyl group of PyA and the minus charges of glutamic acid and aspartic acid of HSA.

MIP, NIP, and MPC were formed by the following procedure. The SiN surface was treated with concentrated ozone in a UV-O 3 cleaner (Samco, UV-1) for 30 min to form hydroxyl groups. The DC chips were placed in a tray containing APTES (1.0 wt\%) dissolved in an ethanol:water mixture $(95: 5 \mathrm{v} / \mathrm{v})$. After 60 min of incubation in an autoclave, the DC chips were rinsed with ethanol and water and heated on a hotplate for $60 \mathrm{~min}$ at $80{ }^{\circ} \mathrm{C}$. The chips were then incubated in a DMF solution containing 2-bromoisobutyric acid (5 mM), NHS (7.5 mM), and EDS·HCl $(7.5 \mathrm{mM})$ for $12 \mathrm{~h}$ and rinsed with DMF to introduce bromo groups to the surface. Then, each polymer layer was formed with SI-AGET ATRP. Materials used for the polymerization were $\mathrm{CuBr}_{2}$, L-ascorbic acid, Bpy (initiator), reducing agent, and metal catalysts.

To form MIP, the DC chips were placed in a sodium phosphate-buffered saline (PBS) ( $\mathrm{pH}$ 7.4) solution containing HSA $(0.1 \mathrm{mM})$, PyA $(0.3 \mathrm{mM})$, MPC $(18 \mathrm{mM})$, MBAA $(0.2 \mathrm{mM})$, copper (II) bromide $\left(\mathrm{CuBr}_{2}\right)(1 \mathrm{mM})$, Bpy $(2 \mathrm{mM})$, and L-ascorbic acid $(0.5 \mathrm{mM})$, purged with nitrogen gas, and incubated for $1 \mathrm{~h}$ at $40{ }^{\circ} \mathrm{C}$. The chips were then washed with ethanol and immersed in ethylenediaminetetraacetic acid tetrasodium salt tetrahydrate (EDTA-4Na) $(1.0 \mathrm{M})$ for 1 $\mathrm{h}$ at room temperature to remove copper (II). After washing with methanol, the chips were immersed in a $1.0 \mathrm{M} \mathrm{NaCl}$ solution containing $\mathrm{SDS}(0.5 \mathrm{wt} \%)$ for $12 \mathrm{~h}$ at room temperature to remove HSA. NIP was also prepared following the above-mentioned procedure without HSA. MPC was prepared without HSA, PyA, or MBAA. 


\subsection{Detection principle}

The DC sensor detects the output intensity of lights emitted from the two ends, $s_{1}$ and $s_{2}$ [Fig. 2(b)]. Normalized outputs $I_{1}$ and $I_{2}$ are given by the following equation.

$$
I_{i}=\frac{s_{i}}{s_{1}+s_{2}} \quad(i=1,2)
$$

$I_{1}$ and $I_{2}$ change through the interaction of propagating lights at the sensing region that accompanies periodic energy transfer between the two parallel waveguides. Here, the distance needed for optical energy to transfer from one waveguide to the other is called the coupling length, $L_{c}$ [Fig. 2(c)]. The binding of the HSA to the MIP formed on the DC surface causes a change in the refractive index. Accompanying this, the output signals $s_{1}$ and $s_{2}$ change with a shift in $L_{c}$. The distribution of light intensity along the sensing region $I_{2}(z)$ is obtained by the coupled-mode theory as follows.

$$
I_{2}(z)=\left(\sin \frac{\pi z}{2 L_{c}}\right)^{2}
$$

In regions where waveguides are separated, no interaction occurs and the distribution of $I_{2}(z)$ is maintained. Therefore, $L_{c}$ can be calculated from Eqs. (1) and (2) using the intensities $s_{1}, s_{2}$ measured at the ends of the waveguides. It is known that there is a linear relationship between the refractive index of the bound protein layer and $L_{c}{ }^{(15,16)}$ From this relation, the concentration of the protein can be known.

\subsection{Experimental setup and characterization}

SEM images of the DC were taken using a field-emission scanning electron microscope (FESEM SU-8020, Hitach). The mold for the PDMS microfluidic channel $(0.8 \mu \mathrm{m}$ wide and 200 $\mu \mathrm{m}$ high) was formed with SU-8 25 and the height was measured with a laser microscope (VK-8500, Keyence). Through-holes (1 mm diameter) were formed by punching to form the solution inlet and outlet. The flow channel and the DC sensing region were aligned under the microscope. The DC chip was fixed on a 6-axis translation stage (OptoSigma). Transverse-electric (TE) polarized light from a semiconductor laser (wavelength: $635 \mathrm{~nm}$, power: $5 \mathrm{~mW}$ ) was end-fire coupled into one of the input waveguides through a long-focus objective lens $(50 \times$, focal length: $4 \mathrm{~mm})$. Two signals from the output waveguides were focused by another objective lens $(20 \times$, focal length: $10 \mathrm{~mm}$ ) onto a CCD camera (SK-TC202USB-AT, OptoSigma). ImageJ (NIH) was used to analyze the near-field images and videos. ${ }^{(22)}$ HSA was detected in the PDMS flow channel. PBS ( $\mathrm{pH}$ 7.4) was introduced into the flow channel as a running buffer, and the HSA solution was introduced. A syringe pump (MD-1000/MD-1001, Bioanalytical Systems) was used for this purpose. A 6-port injection valve (V-451, IDEX Health and Science) was used to efficiently switch between PBS and HSA solutions. The values of the baseline and the output signal of the DC sensor were determined by calculating the time average of the fluctuating output signal numerically within a certain period of time. 


\section{Results and Discussion}

\subsection{MIP, NIP, and MPC on the DC sensor}

Figure 3 shows a SEM image of the cross section of the sensitive area of the DC sensor. From this image, it was found that fabrication error was less than $10 \%$, and expected waveguide structures could be obtained reproducibly.

MIP, NIP, and MPC on the DC sensor influence the baseline. To examine this point, $\Delta L_{c}$ was measured after forming the MIP, NIP, and MPC layers. The obtained values were 0.24 , 0.47 , and $0.54 \mu \mathrm{m}$, respectively (Fig. 4). The thickness of each polymer layer $\left(t_{a d}\right)$ can be estimated by electromagnetic simulation for propagating lights. It is known that there is a linear relationship between $L_{c}$ and $t_{a d}$. The values obtained by simulation and experiment agreed well. ${ }^{(15)}$ On the basis of the results, it was confirmed that the MIP, NIP, and MPC layers actually existed on the DC; the calculated thicknesses of the layers were $3.3,6.4$, and $7.3 \mathrm{~nm}$, respectively. The calculated thicknesses were less than those of polymer layers prepared previously with the same material and procedure. ${ }^{(19)}$ This discrepancy was possibly caused by the difference in the structure of the surface. In our DC, there is a very narrow gap in the sensing region, which may have affected the polymerization reaction and resulted in small $t_{a d}$.

\subsection{Detection of HSA}

The binding of HSA to MIP, NIP, and MPC layers on the DC sensing region was examined. Figure 5 shows the change in $\Delta L_{c}$ while HSA was binding to MIP and NIP on the DC sensor surface. Here, the baseline was first recorded in a continuous PBS flow at $20 \mu \mathrm{L} / \mathrm{min}$. Then, a HSA solution was introduced into the flow channel at $20 \mu \mathrm{L} / \mathrm{min}$. A distinct change from the baseline was observed upon the replacement to the HSA solution. Figure 6 shows $\Delta L_{c}$ recorded with MIP, NIP, and MPC for $1.0 \mu \mathrm{M}$ HSA. With MIP, NIP, and MPC, $\Delta L_{c}$ to $1.0 \mu \mathrm{M}$ HSA

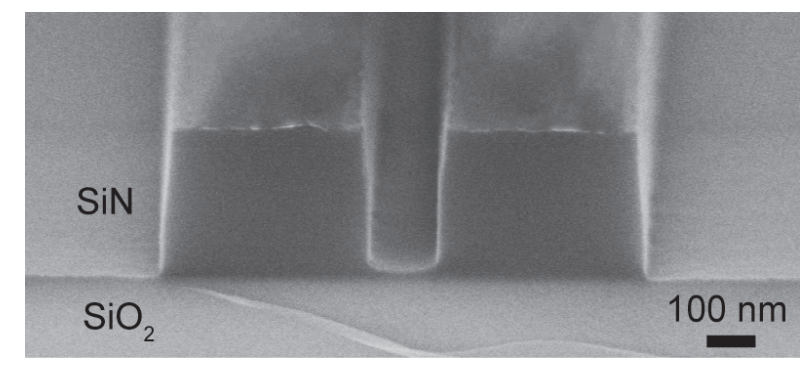

Fig. 3. SEM image of the cross section of the sensitive area of the DC sensor.

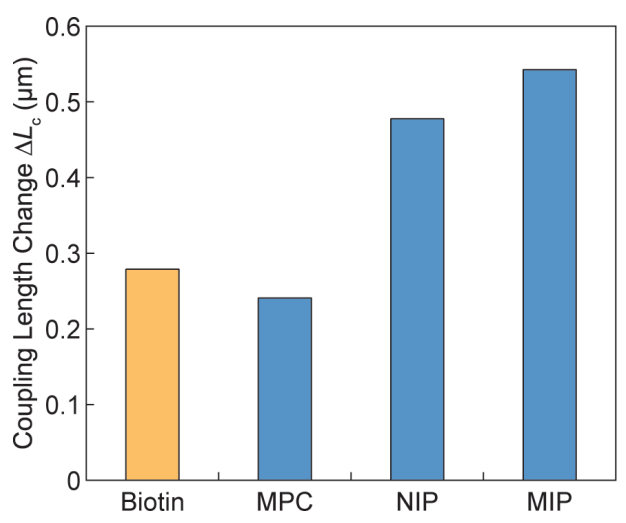

Fig. 4. (Color online) baseline detection with PBS making contact with sensing region surface of biotin-, MPC-, NIP-, and MIP-immobilized DC sensors. 


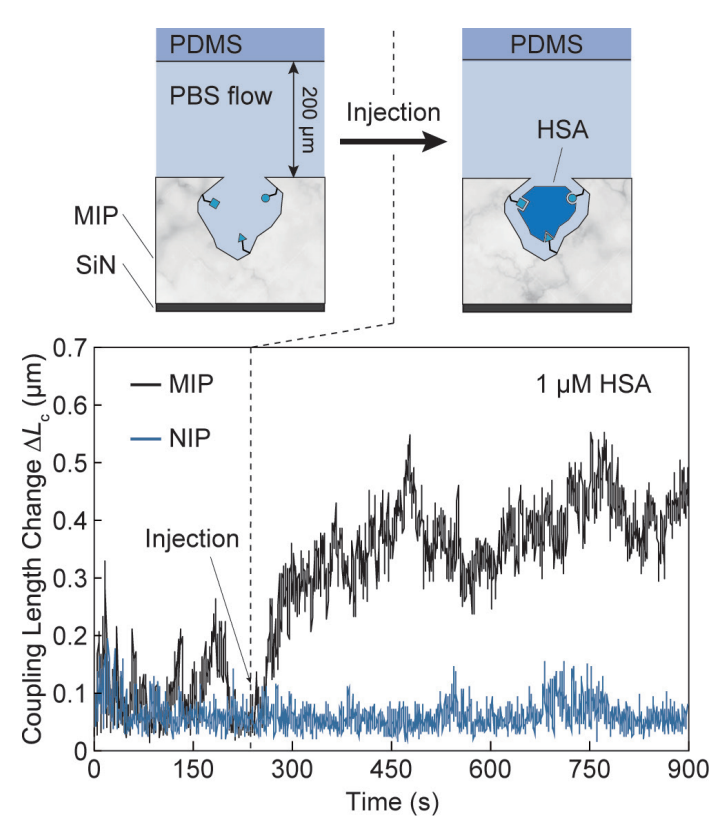

Fig. 5. (Color online) $\Delta L_{c}$ observed with MIP and NIP upon the addition of HSA $(1.0 \mu \mathrm{M})$.

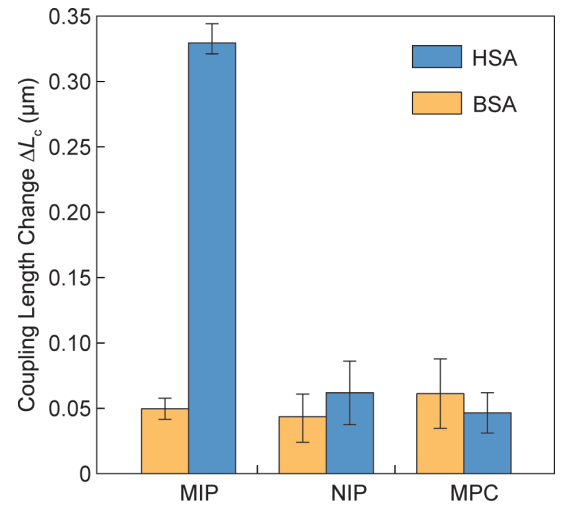

Fig. 6. (Color online) $\Delta L_{c}$ observed with MIP, NIP, and MPC upon the addition of HSA $(1.0 \mu \mathrm{M})$ and $\operatorname{BSA}(1.0 \mu \mathrm{M})$.

was $0.33,0.06$, and $0.05 \mu \mathrm{m}$ on average, respectively, indicating that HSA bound to only MIP selectively. For comparison, the response to $1.0 \mu \mathrm{M}$ BSA was also checked with MIP, NIP, and MPC. In these cases, $\Delta L_{c}$ was $0.05,0.04$, and $0.06 \mu \mathrm{m}$, respectively, which are comparable to the responses to HSA with NIP and MPC. The results indicate the selective binding of HSA to the MIP layer.

With MIP, a linear relationship was observed for HSA concentrations lower than $1.0 \mu \mathrm{M}$ and the response tended to saturate at higher concentrations. With reference to the response profile shown in Fig. 5, the fluctuation of the baseline is estimated as $0.13 \mu \mathrm{m}$. If the lower detection limit is defined as the concentration corresponding to twice this value, it is $0.26 \mu \mathrm{M}$.

\subsection{Regeneration of the sensor and reproducibility of the response}

The response of the DC sensor with MIP after the regeneration of MIP was investigated by three sequential measurements. After one measurement, MIP was regenerated by immersing the chip in a $1.0 \mathrm{M} \mathrm{NaCl}$ solution containing SDS $(0.5 \mathrm{wt} \%)$ for $12 \mathrm{~h}$ at room temperature. Figure 7 shows the change in $\Delta L_{c}$ obtained with the same sensor after the three cycles of adsorption and regeneration. For $1.0 \mu \mathrm{M} \mathrm{HSA}, \Delta L_{c}$ observed in the three measurements was 0.32 , 0.34 , and $0.34 \mu \mathrm{m}$, respectively, which are very similar values. The result suggests that MIP was actually regenerated and the sensor could be used repeatedly. 

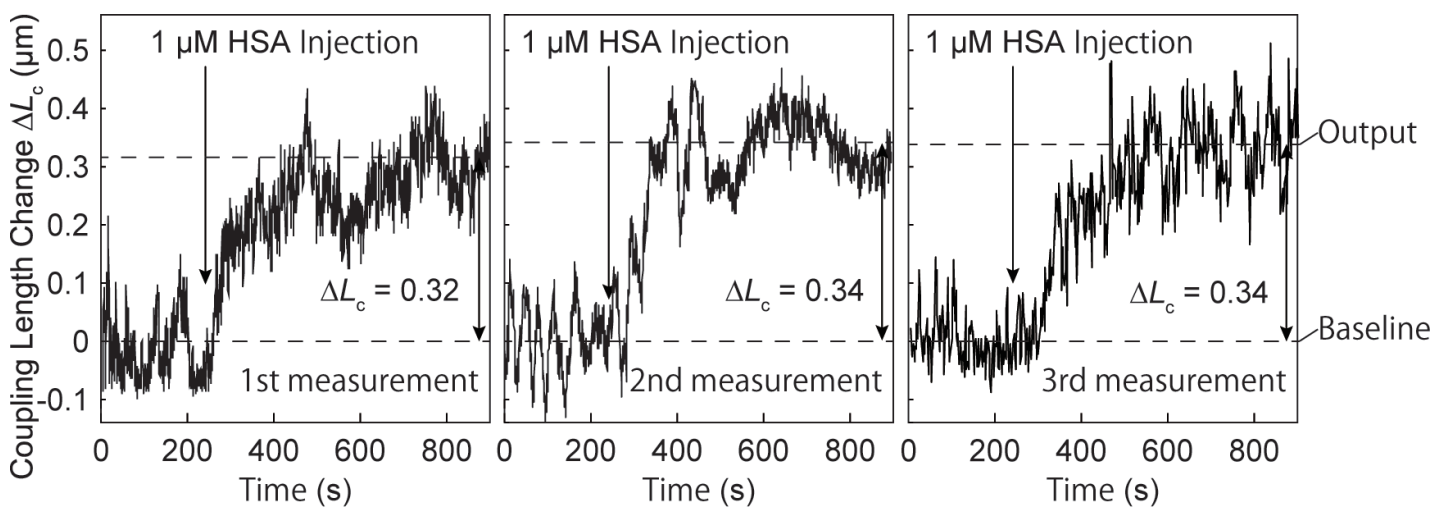

Fig. 7. Response of the same sensor to $1.0 \mu \mathrm{M}$ HSA. The measurements were conducted 3 times. Dotted lines in each graph represent the baseline and the output to HSA obtained by calculating time average of the fluctuating output signals numerically between $0-200$ and $700-900$ s, respectively.

\section{Conclusions}

A DC sensor coupled with MIP can be used for the detection of proteins, as was actually demonstrated using HSA as a model analyte. Selectivity of the sensor was excellent. Distinct changes were observed in $L_{c}$ accompanying the binding of HSA to MIP. On the other hand, no response was observed with BSA, NIP, or MPC as the sensing layer. MIP can be regenerated. The responses after the regeneration were very similar.

\section{Acknowledgments}

We thank Dr. Hirotaka Osato of National Institute of Material Science and Professor Edwin T. Carlen of University of Tsukuba for their suggestions and support. K. U. thanks the JSPS Research Fellowships for Young Scientists (Project No. 16J05520) for financial support. The devices were fabricated with the support of University of Tsukuba Nanotechnology Platform and the National Institute of Material Science Nanofabrication Platform.

\section{References}

R. Datar, S. Kim, S. Jeon, P. Hesketh, S. Manalis, A. Boisen, and T. Thundat: MRS Bull. 34 (2009) 449.

2 D. Grieshaber, R. Mackenzie, J. Vörös, and E. Reimhult: Sensors 8 (2008) 1400.

3 Z. Shen, M. Huang, C. Xiao, Y. Zhang, X. Zeng, and P. G. Wang: Anal. Chem. 79 (2008) 2312.

4 F. Vollmer and L. Yang: Nanophotonics 1 (2012) 267.

5 X. Guo: J. Biophotonics 5 (2012) 483.

6 M. C. Estevez, M. A. Otte, B. Sepulveda, and L. M. Lechuga: Anal. Chim. Acta 806 (2014) 55.

7 F. Prieto, B. Sepúlveda, A. Llobera, C. Domínguez, A. Abad, A. Montoya, and L. M. Lechuga: Nanotechnology 14 (2003) 907.

8 A. Ymeti, J. S. Kanger, R. Wijn, P. V. Lambeck, and J. Greve: Sens. Actuators, B 83 (2002) 1.

9 M. C. Estevez, M. Alvarez, and L. M. Lechuga: Laser Photon. Rev. 6 (2012) 463.

10 P. Kozma, F. Kehl, E. Ehrentreich-Förster, C. Stamm, and F. F. Bier: Biosens. Bioelectron. 58 (2014) 287.

11 H. Sayama and T. Takeuchi: Kobunshi Ronbunshu 73 (2016) 19 (in Japanese).

12 R. Schirhagl: Anal. Chem. 86 (2014) 250. 
13 G. Vasapollo, R. D. Sole, L. Mergola, M. R. Lazzoi, A. Scardino, S. Scorrano, and G. Mele: Int. J. Mol. Sci. 12 (2011) 5908.

14 K. Uchiyamada, K. Okubo, M. Yokokawa, E. T. Carlen, K. Asakawa, and H. Suzuki: Opt. Express 23 (2015) 17156.

15 K. Okubo, K. Uchiyamada, K. Asakawa, and H. Suzuki: Opt. Eng. 56 (2017) 017101.

16 G. J. Quinlan, G. S. Martin, and T. W. Evans: Hepatology 41 (2005) 1211.

17 C. Dai, J. Zhang, X. Zhou, and S. Liu: PLOS ONE 8 (2013) e78167.

18 T. Nagata, T. Tanaka, K. Miyake, H. Kurotaki, S. Yokoyama, and M. Koyanagi: Jpn. J. Appl. Phys. 33 (1994) 822.

19 Y. Innoue, A. Kuwahara, K. Ohmori, H. Sunayama, T. Ooya, and T. Takeuchi: Biosens. Bioelectron. 48 (2013) 113.

20 K. Min, H. Geo, and K. Matyjaszewski: J. Am. Chem. Soc. 127 (2005) 3825.

21 K. Ishihara, T. Ueda, and N. Nakabayashi: Polym. J. 22 (1990) 355.

22 C. A. Schneider, W. S. Rasband, and K. W. Eliceiri: Nat. Methods 9 (2012) 671. 